Unter dem Interleukin-12/23-Inhibitor Stelara ${ }^{\circledR}$ (Ustekinumab) konnte im Vergleich zu den TNFalpha-Inhibitoren Adalimumab und Etanercept eine höhere Persistenz $z^{\star}$ gezeigt werden. Eine kontinuierliche Therapie ist grundlegend für den Erfolg einer Behandlung, jedoch brechen ungefähr 20-30\% der Psoriasis-Patienten, die mit einem TNF-alpha-Inhibitor behandelt werden, die Therapie aufgrund von Wirkungslosigkeit oder Nebenwirkungen ab [1]. «Für die langfristige Krankheitskontrolle benötigen wir Präparate mit guter Langzeitwirksamkeit und -verträglichkeit - Eigenschaften, die sich in einer guten Therapietreue widerspiegeln sollten", so der Dermatologe Prof. Dr. Kristian Reich, Hamburg.

In einer Studie auf Basis von Daten aus dem dänischen Patientenregister Dermbio haben Anders Clemmensen und seine Kollegen über einen Zeitraum von 321 Tagen die Persistenz und die Wirksamkeit von Ustekinumab bei Patienten mit Plaque-Psoriasis anhand eines PASI*-75-Ansprechens untersucht. Von den 71 Patienten, die mit Ustekinumab behandelt wurden, waren 24 anti-TNF-alpha-naive und 47 anti-TNF-alpha-erfahrene Patienten. Diese wurden mit einer Kontrollgruppe von insgesamt 108 Plaque-Psoriasis-Patienten verglichen, die zur gleichen Zeit mit Adalimumab oder Etanercept behandelt wurden und ebenfalls entweder anti-TNF-alpha-naiv $(\mathrm{n}=77)$ oder -erfahren $(n=31)$ waren [1]. Im Vergleich $\mathrm{zu}$ den TNF-alpha-Inhibitoren Adalimumab und Etanercept konnte unter Ustekinumab eine bessere Persistenz nach einem Jahr gezeigt werden. Über den Beobachtungszeitraum von 321 Tagen haben 3 der Ustekinumab-Patienten (4,2\%) die Therapie abgebrochen. Davon brachen 2 Patienten die Therapie wegen unerwünschter Er- eignisse $a b$ und ein weiterer Patient erschien nicht mehr zu den Nachuntersuchungen. Dagegen brachen insgesamt 21 Patienten (19,4\%), die Adalimumab oder Etanercept erhielten, die Therapie wegen mangelnder Wirksamkeit ab, 5 Patienten (4,6\%) wegen unerwünschter Ereignisse und 3 (2,7\%) aus anderen Gründen. Die daraus resultierenden Persistenzraten von Ustekinumab und dieser TNF-alpha-Inhibitoren unterschieden sich signifikant $(\mathrm{p}<0,001)$. Somit zeigte Ustekinumab im Vergleich eine bessere Persistenz nach einem Jahr (95,8 vs. $73,3 \%$ ) [1]. Wie wichtig eine kontinuierliche Therapie ist, bestätigt auch Reich: «Bei einer Monotherapie mit Biologika kann eine intermittierende Behandlung das Risiko der Bildung von Anti-Drug-Antikörpern erhöhen. Medizinisch nicht erforderliche Therapiepausen und Therapiewechsel sind für Arzt und Patient mit einem erhöhten zeitlichen Aufwand und einer oft schlechteren Krankheitskontrolle verbunden.»

In der Studie von Clemmensen wurde auch die Wirksamkeit von Ustekinumab untersucht. Ein PASI 75-Ansprechen erreichten 80\% der Ustekinumab-Patienten nach einer durchschnittlichen Zeit von 112 Tagen. Ein vorheriges Nichtansprechen auf ein oder mehrere TNFalpha-Inhibitoren beeinflusste das PASI-75-Ansprechen in der Ustekinumab-Gruppe nicht. In Verbindung mit der Persistenz bewerteten die Autoren der Studie die klinische Wirksamkeit von Ustekinumab als ausgezeichnet [1].

Im Rahmen der gemeinsamen Jahrestagung des American College of Rheumatology (ACR) und der Association of Rheumatology Health Professionals (ARHP) 2014 haben A. Menter und
Kollegen bei der Präsentation eines Posters, das die Ergebnisse des internationalen Registers «Psoriasis Longitudinal Assessment and Registry» (PSOLAR) beinhaltete, ebenfalls noch einmal die signifikant bessere Persistenz unter Ustekinumab im Vergleich zu Anti-TNF-alphaTherapien in der Behandlung von Plaque-Psoriasis bestätigt [2]: Biologikanaive Patienten zeigten demnach eine signifikant bessere Persistenz unter Ustekinumab im Vergleich zu allen anderen Biologika (Infliximab vs. Ustekinumab: $\mathrm{p}=0,0003$; Adalimumab vs. Ustekinumab: $p<0,0001$; Etanercept vs. Ustekinumab: $\mathrm{p}<0,0001)$. Dementsprechend war bei einer Biologika-Erstlinienanwendung die Wahrscheinlichkeit einer kontinuierlichen Therapie (gemessen als Hazard Ratio (HR)) deutlich erhöht, wenn anstatt mit einem TNF-alpha-Inhibitor mit Ustekinumab behandelt wurde (Infliximab vs. Ustekinumab: HR 3,04 (1,66-5,57); Adalimumab vs. Ustekinumab: HR 4,99 (3,397,35); Etanercept vs. Ustekinumab: HR 5,59 $(3,77-8,29))$.

${ }^{\star}$ PASI $=$ Psoriasis Area and Severiti Index

${ }^{*}$ Persistenz $=$ Das kontinuierliche Fortführen einer Therapie bei einem chronisch kranken Patienten, der mit einem bestimmten Medikament therapiert wird.

Literatur

1 Clemmensen A et al.: J Eur Acad Dermatol Venerol 2011;25:1037-1040.

2 Menter A et al.: Arthritis Rheumatol 2014;66(suppl 10): S693, abstr 1569

Weitere Informationen bei

FleishmanHillard Germany

Marthe Nienhuys

Marthe.Nienhuys@fleishmaneurope.com

\title{
PharmaTicker+++ PharmaTicker+++ PharmaTicker+++ PharmaTicker+++
}

LEO Pharma. Mit Picato ${ }^{\circledR}$ (Ingenolmebutat) ist seit November 2012 ein Präparat zur topischen Behandlung von nichthyperkeratotischen, nichthypertrophen aktinischen Keratosen bei Erwachsenen zugelassen. Im Vergleich zu anderen Behandlungsoptionen, die über Wochen oder Monate angewendet werden müssen, liegt der besondere Vorteil der topischen Therapie mit Ingenolmebutat in der kurzen Anwendungsdauer von 1-3 Tagen. Die sehr kurze Therapiedauer geht mit einer Adhärenzrate von mehr als $98 \%$ einher.

m:werk GmbH \& Co. KG

Gunnar Lühring

luehring@m-werk.de
Celgene. Im Januar 2015 erhielt OTEZLA ${ }^{\circledR}$ (Apremilast), ein oral verabreichbarer niedermolekularer Inhibitor des Enzyms Phosphodiesterase-4 (PDE4), die Marktzulassung in der Europäischen Union zur Behandlung der mittelschweren bis schweren chronischen Plaque-Psoriasis sowie der aktiven Psoriasis-Arthritis bei erwachsenen Patienten. Der PDE4-Inhibitor zeigt eine überzeugende Wirksamkeit auf Haut, Kopfhaut, Nägel, Gelenke und Juckreiz und ist ab sofort auf dem deutschen Markt verfügbar. Celgene GmbH
info@ celgene.de
www.celgene.de
Merz Aesthetics erweitert mit dem integrierten Lokalanästhetikum in Belotero ${ }^{\circledR}$ Volume Lidocaine sein innovatives Belotero ${ }^{\circledR}$-Sortiment und bietet durch den Zusatz von Lidocain $(0,3 \%)$ einen noch höheren Patientenkomfort, um verlorenes Gesichtsvolumen wieder herzustellen. Der speziell formulierte Volumen-Filler mit außergewöhnlichen Modelliereigenschaften kombiniert einen extra hohen Hyalurongehalt $(26 \mathrm{mg} / \mathrm{ml}$ Hyaluronsäure) mit patentierter $\mathrm{CPM}^{\circledR}$-Technologie und langanhaltender Wirkung.

Merz Pharmaceuticals GmbH

Karin Tauber

karin.tauber@merz.de 


\section{PharmaNews}

Karger Kompass Dermatologie 3 | 1 | 15

\section{BRAF-Hemmung beim malignen IMelanom - Langzeitansprechen mit Dabrafenilb}

Das maligne Melanom zeigt ein aggressives Ausbreitungsverhalten unter anderem in das Gehirn und zeigt häufig eine Resistenz gegenüber einer Chemo- und Strahlentherapie. Bis 2011 hatten Patienten mit Fernmetastasierung eine mediane Überlebenszeit von 9-12 Monaten [1]; Heilung konnte laut Prof. Carola Berking, München, nur bei 1-2\% der Patienten erzielt werden. Mit einer palliativen Chemotherapie, der seit 1975 einzigen zugelassenen Therapieoption für Patienten mit einem metastasierten Melanom, wurden Ansprechraten von 5-25\% erreicht, so Berking. 2011 wurde Vemurafenib als erster BRAF-Inhibitor beim metastasierten malignen Melanom von der FDA und 2012 von der EMA zugelassen [2]. Im August 2013 erfolgte die europäische Zulassung des 2. BRAF-Inhibitors Dabrafenib als Monotherapie zur Behandlung des inope- rablen oder metastasierten $B R A F^{V 600}$-mutierten malignen Melanoms. In der BREAK-3-Studie war mit Dabrafenib bei 59\% der Patienten ein Tumoransprechen $(\mathrm{CR}+\mathrm{PR})$ mit einer medianen Dauer von 8,0 Monaten beobachtet worden, im Kontrollarm mit Dacarbazin-Chemotherapie sprachen nur 24\% mit einer medianen Dauer von 7,6 Monaten an. Das mediane PFS betrug in einer Auswertung nach 10,5 bzw. 9,9 Monaten Nachbeobachtungszeit 6,9 Monate für Patienten im Dabrafenib-Arm und 2,7 Monate unter Dacarbazin (HR 0,37; p < 0,0001) [3]. «Für Dabrafenib haben wir inzwischen Langzeitdaten. Das mediane Gesamtüberleben liegt jetzt schon bei 20,1 Monaten. $31 \%$ der in die Studie eingeschlossenen Patienten überlebten 3 Jahre. Früher lag das 3-Jahres-Gesamtüberleben im Bereich von 5\%», sagte Berking. In der BREAK-MB-Studie wurde bei Melanompatienten mit $B R A F^{V 600 E}$-Mutation und Hirnmetastasen gezeigt, dass Dabrafenib auch bei Hirnmetastasierung, einem häufigen Problem beim malignen Melanom, wirksam ist [4].

\section{Quelle}

Fachpresse-Workshop der POMME-med GmbH am 18. Dezember 2014 in München

Literatur

1 Balch CM et al.: J Clin Oncol 2009;27:6199-6206

2 Chapman PB et al.: N Engl J Med 2011;364:2507-2516.

3 Hauschild A et al.: J Clin Oncol 2013;31:abstr 9013.

4 Long GV et al.: Lancet Oncol 2012;13:1087-1095.

Weitere Informationen bei

POMME-med GmbH

Carina Jung

jung@pomme-med.de

\section{Erivedge ${ }^{\circledR}:$ Komplette Remission bei jedem dritten Patienten mit fortgeschrittenem Basalzellkarzinom}

Einen weiteren Beleg für die gute klinische Wirksamkeit und Sicherheit von Erivedge ${ }^{\otimes}$ (Vismodegib) liefert eine aktuelle Interimsanalyse der globalen Sicherheitsstudie Safety Events in Vismodegib (STEVIE) mit Behandlungsdaten von mittlerweile 500 Patienten. Das zentrale Ergebnis: Etwa 9 von 10 Patienten mit fortgeschrittenem Basalzellkarzinom (BCC) sprechen auf die Behandlung mit Vismodegib an - bei etwa jedem 3. Patienten wird sogar eine komplette Remission erreicht. Die Verträglichkeit entspricht dem bekannten Sicherheitsprofil des Wirkstoffs, so die beim Kongress der Society for Melanoma Research (SMR) im November 2014 in Zürich präsentierten Daten.

Dem Hedgehog-Signalweg kommt eine Schlüsselrolle bei der Pathogenese des BCC zu. Dass sich die Hemmung des Signalwegs durch Vis- modegib in beeindruckenden Therapieerfolgen niederschlägt, zeigt die jüngste Interimsanalyse der Studie STEVIE, in der die Daten von 500 Patienten mit mindestens 1-jährigem Follow-up ausgewertet wurden [1]. Primärer Endpunkt der offenen, multizentrischen Studie ist die Sicherheit der Medikation, sekundäre Endpunkte sind unter anderem die Ansprechrate, die Ansprechdauer, die Lebensqualität unter der Therapie und das Auftreten von Krankheitssymptomen.

Die aktuell auf dem SMR-Kongress präsentierte Interimsanalyse der STEVIE-Studie zeigt, dass insgesamt 91,6\% der Patienten auf die Behandlung mit Vismodegib ansprechen. Mit $32,2 \%$ war die Rate der Patienten mit komplettem Ansprechen außerordentlich hoch. Bei weiteren 32,8\% der Studienteilnehmer zeigte sich eine partielle Remission und bei 26,6\% eine Krankheitsstabilisierung. Anhand einer Fotodokumentation wurde eine beeindruckende Tumorschrumpfung unter Vismodegib demonstriert. Die Zeit bis zur Response lag in der Studie bei median 2,7 Monaten; die Ansprechdauer betrug median 22,7 Monate. Für das PFS wurde ein Median von 20,2 Monaten ermittelt.

Literatur

1 Dréno $B$ et al.: Posterpräsentation, SMR-Kongress, November 2014, Zürich.

Weitere Informationen bei

Roche Pharma AG

Ulla Satzger

ulla.satzger@roche.com

\section{PharmaTicker+++ PharmaTicker+++ PharmaTicker+++ PharmaTicker+++}

AbbVie. Der Ausschuss für Humanarzneimittel der Europäischen Arzneimittel-Agentur (EMA) hat die Zulassungserweiterung für Adalimumab $\left(\right.$ Humira $\left.^{\circledR}\right)$ zur Behandlung schwerer chronischer Plaque-Psoriasis bei Kindern und Jugendlichen ab 4 Jahren empfohlen. Die Empfehlung zur Zulassungserweiterung (positive opinion) basiert auf den Ergebnissen einer Phase-III-Studie, die auf einem der kommenden medizinischen Kongresse vorgestellt werden.

AbbVie Deutschland GmbH \& Co. KG Jana Umbreit

Jana.Umbreit@abbvie.com
Galderma. Die erstmals beim $12^{\text {th }}$ EADV Spring Symposium und AAD $73^{\text {rd }}$ Annual Meeting als Poster präsentierten Ergebnisse der ELANGStudie bestätigen die starke Wirksamkeit von Epiduo ${ }^{\circledR}$ in der Langzeitbehandlung der mittelschweren und schweren Akne in der täglichen Praxis. Über den 9-monatigen Studienverlauf mit über 5000 Patienten verbesserte sich die Akne von einem mittleren zu einem leichten Schweregrad, wobei $25,8 \%$ der Patienten am Studienende frei von Akne-Läsionen waren.

Galderma Laboratorium GmbH

Dr. Doris Porombka

doris.porombka@galderma.com
Mundipharma. Mit Remsima ${ }^{\circledR}$ steht das erste Biosimilar des TNF-alpha-Blockers Infliximab zur Verfügung. Die Ergebnisse der Zulassungsstudien haben gezeigt, dass Remsima ${ }^{\circledR}$ sowohl bezüglich pharmakokinetischer Parameter, wie der Bioverfügbarkeit, als auch in den klinischen Parametern Wirksamkeit und Sicherheit eine hohe Ähnlichkeit mit Remicade ${ }^{\circledR}$ aufweist. Die EMA hat das Biosimilar daher für alle Indikationen zugelassen, in denen auch das Referenzpräparat zugelassen ist - z.B. auch für Psoriasis-Arthritis und Psoriasis.

Mundipharma Deutschland GmbH \& Co. KG Felicitas Speranza

felicitas.speranza@mundipharma.de 


\section{PharmaNews}

Karger Kompass Dermatologie 3 | 1 | 15

Cosenty ${ }^{\circledR}$ erhält' als erster IL-17A-Inhibitor die EU-Zulassung für die Erstlinientherapie bei mittelschwerer bis schwerer Psoriasis

Januar 2015 hat die Europäische Kommission die Zulassung für Cosentyx ${ }^{\circledR}$ (Secukinumab, vormals AIN457) als systemische Erstlinientherapie von mittelschwerer bis schwerer Plaque-Psoriasis bei Erwachsenen, die für eine systemische Therapie in Frage kommen, erteilt

Secukinumab (in einer Dosis von $300 \mathrm{mg}$ ) ist damit der 1. Interleukin(IL)-17A-Inhibitor, der in Europa die Marktzulassung erhalten hat. Mit diesem Biologikum steht nun eine neuartige und wichtige Option für die betroffenen Patienten zur Verfügung. Die anderen zurzeit verfügbaren Biologika zur Behandlung von Psoriasis - Tumornekrosefaktor(TNF)-Blocker und der IL-12/23-Inhibitor - werden im Gegensatz dazu in Europa als systemische Zweitlinientherapie empfohlen [1-4].
In klinischen Studien erzielten mindestens 70\% der Patienten unter $300 \mathrm{mg}$ Secukinumab während der ersten 16 Wochen der Behandlung eine symptomfreie Haut (PASI 100) oder fast symptomfreie Haut (PASI 90) [5]. Diese Verbesserung konnte bei der Mehrheit der Patienten, die die Behandlung fortsetzten, bis zur 52. Woche aufrechterhalten werden [5]. In der zulassungsrelevanten FIXTURE-Studie konnte ein schnelles Wirkansprechen bei den Patienten unter Secukinumab $300 \mathrm{mg}$ beobachtet werden. Deutliche Verbesserungen wurden bereits nach 2 Wochen bemerkt [5]. Die Daten des klinischen Studienprogramms zu Secukinumab zeigten auch einen signifikanten positiven $\mathrm{Zu}$ sammenhang zwischen dem Erreichen einer symptomfreien oder nahezu symptomfreien
Haut und der gesundheitsbezogenen Lebensqualität der Psoriasis-Patienten [6].

Literatur

1 Mohanan S et al.: Journal of Dermatological Treatment 2014;25:50-53.

2 www.ema.europa.eu/docs/en_GB/document_library/EPAR Product_Information/human/000262/WC500027361.pdf (letzter Zugriff November 2014).

3 www.ema.europa.eu/docs/en GB/document library/EPAR Product_Information/human/000481/WC500050870.pdf (letzter Zugriff November2014).

4 www.ema.europa.eu/docs/en_GB/document_library/EPAR Product_Information/human/000958/WC500058513.pdf (letzter Zugriff November 2014).

5 Langley RG et al.: N Engl J Med 2014;371:326-338.

6 McLeod LD et al.: European Association of Dermatology and Venereology, Amsterdam, Niederlande, 2014: Poster 1681.

Weitere Informationen bei

Novartis Pharma GmbH

Diana Drescher

diana.drescher@novartis.com

\section{FDA-Zulassung für Pembrolizumab beim malignen Melanom}

Der humanisierte PD-1-Antikörper Pembrolizumab wurde in der Phase-I-Studie KEYNOTE001 bei 190 Ipilimumab-naiven und 221 Ipilimumab-vorbehandelten Patienten mit malignem Melanom in den Dosierungen $10 \mathrm{mg} / \mathrm{kg}$ q2w $(\mathrm{n}=57), 10 \mathrm{mg} / \mathrm{kg} \mathrm{q3} \mathrm{w}(\mathrm{n}=192)$ oder $2 \mathrm{mg} / \mathrm{kg}$ $\mathrm{q} 3 \mathrm{w}(\mathrm{n}=162)$ überprüft [1]. Insgesamt waren $56 \%$ der Tumoren im AJCC-Stadium M1c, 8\% der Patienten wiesen Hirnmetastasen auf, 77\% waren vortherapiert und 35\% hatten einen erhöhten LDH-Wert. Ein Ansprechen auf die Therapie nach RECIST-Kriterien zeigten $44 \%$ der Behandlungs-naiven und 28\% der Ipilimumabvorbehandelten Patienten. 88\% der Patienten waren zur Zeit der Zwischenauswertung in anhaltender Remission, wie Prof. Axel Hauschild, Kiel, ausführte. Das mediane Überleben war noch nicht erreicht. Nur bei $12 \%$ der Patienten traten Nebenwirkungen der Grade 3/4 auf. Die amerikanische Zulassungsbehörde FDA hat Pembrolizumab aufgrund dieser Ergebnisse unter dem Handelsnamen Keytruda ${ }^{\circledR}$ die USZulassung erteilt. Ein weiterer PD-1-Antikörper, Nivolumab, zeigte vergleichbare Ergebnisse mit Überlebensraten von $63 \%$ nach einem Jahr, $48 \%$ nach 2 Jahren und $41 \%$ nach 3 Jahren. Damit sei für beide Anti-PD-1-Strategien eine schnelle und gute Remission mit langer Dauer gezeigt worden, auch wenn die Nachbeobachtungszeiten noch relativ kurz seien, so Hauschild. Er erwarte eine deutliche Überlebensverlängerung gegenüber den Standards, aber Ergebnisse der Phase-III-Studien zum Gesamtüberleben stünden noch aus.

«Die immunbiologische Therapie des malignen Melanoms ist eine andauernde Erfolgsgeschich- te», begeisterte sich Hauschild. Sie begann beim ASCO-Kongress 2012, wo für Nivolumab in einer Phase-I-Studie eine herausragende Wirkung bei Melanom, Nieren- und Bronchialkarzinom gezeigt wurde. Beim ASCO 2013 wurden dann die Daten zu Pembrolizumab und zur Kombination Nivolumab plus Ipilimumab vorgestellt. Beim ASCO 2014 wurden zu allen Studien Updates präsentiert.

\section{Quelle}

Fachpresse-Workshop der POMME-med GmbH am 6. November 2014 in München

Literatur

1 Ribas A et al.: J Clin Oncol 2014;32:5s:abstr LBA9000.

Weitere Informationen bei

POMME-med GmbH

Carina Jung

jung@pomme-med.de

Konfokale Mikroskopie setzt sich in den Praxen durch

Seit über 10 Jahren VivaScope ${ }^{\circledR}$ in Europa - eine innovative Methode etabliert sich

Das VivaScope liefert nichtinvasiv Schichtbilder bis zur oberen Dermis in zellulärer Auflösung. Die etwa 10-minütige Untersuchung wird von den med. Fachangestellten übernommen. Arzt und Patient haben sofortige Gewissheit darüber, wie die Läsion weiter behandelt wird. Durch ein effizientes, kostenloses Trainingsprogramm ist der Dermatologe schnell in der Lage, eine präzise Diagnose zu stellen. Über 537 Publikationen, Leitlinien und Lehrbücher geben zudem hohe Sicherheit. Die Abstracts zur konfokalen Laserscan-Mikroskopie (KLSM) werden regelmäßig unter www. vivascope-pub.com zusammengestellt.

In den letzten 10 Jahren konnte die MAVIG GmbH etwa 230 Geräte in Europa installieren, davon allein über 50 in deutschen Praxen. Somit nehmen die niedergelassenen Dermatologen in Deutschland eine internationale Vorreiterrolle ein.
Zurückzuführen ist dies zum einen auf die Sicherheit und Abrechenbarkeit der Methode und zum anderen auf deren Praxistauglichkeit: So kann das VivaScope in die Bildgebungskette von Ganzkörperfotografie und Dermatoskopie integriert werden und Geräte, Betrachtungslizenzen und Schnittstellen zur Praxis-Software können miteinander über die IT-Lösung «VivaLan» vernetzt werden.

Weitere Informationen über das VivaScope unter: www.vivascope.eu.

\section{Mavig GmbH}

VivaScope Systems

Birgit Elisat

elisat@mavig.com 